\title{
Individuals at Risk in Families with Genetic Disease
}

\author{
CHARLES SMITH, SUSAN HOLLOWAY, and ALAN E. H. EMERY
}

From the University Department of Human Genetics, Western General Hospital, Edinburgh

With the control of many infectious diseases and improvement in medical care, there have been dramatic changes in the pattern of mortality and morbidity in society. As a result, genetic diseases have been increased in their relative importance in the population. For example, Roberts, Chavez, and Court (1970) have found in hospital deaths among children that genetic conditions were directly or indirectly involved in over $40 \%$ of cases. Since the liability to genetic disease is inherited and intrinsic to the individual and to his family, rather than acquired or extrinsic as for non-genetic diseases, quite different systems of prevention and control are required. To some extent, these systems will require new departures from the established methods of medical practice.

Until recently the main application of medical genetics has been in counselling the parents of affected children. The possibility of extending the scope for application has been examined recently. Fraser and Motulsky (1968) estimated what proportion of cases of genetic disease might be prevented, and Smith (1970) has examined the value of different routes of prevention and the possibility of a genetic register system. McKusick (1969) has also discussed a medical record system for family follow-up in the detection and early treatment of cases of genetic disease. In a previous study (Emery and Smith, 1970), it was shown that only a small proportion of individuals 'at risk' of having affected children were in fact referred specifically for genetic counselling. Many were referred only after the birth of an affected child which otherwise could have been prevented. These results confirmed the need for a genetic register system in preventing genetic disease.

The object of this paper is to outline our experiences in recording and storing relevant data on families with genetic disease and in assessing risks to various members of the family. Details of the procedure used and a summary and interpretation of the data collected so far are presented.

\section{Methods and Material}

Assessment of Risks. Roberts (1962) grouped genetic diseases into those with a high risk of recurrence (greater than 1 in 10) and those with a low risk of reccurrence (less than 1 in 20). This convention is now generally accepted and is adopted here. Thus, an individual was defined to be 'at risk' if he had a greater than $10 \%$ risk (1) of becoming affected or (2) of having affected children or children who will be 'at risk'. Those unlikely to have children in the future either due to their disease condition or due to their age (over 40 years) are excluded from category 2.

The methods of assessing the risks to family members are best described from some examples (Fig. 1).

In family $A$ with an autosomal recessive (AR) condition, the mother was the first contact. She came for counselling retrospectively, ie, after the birth of her

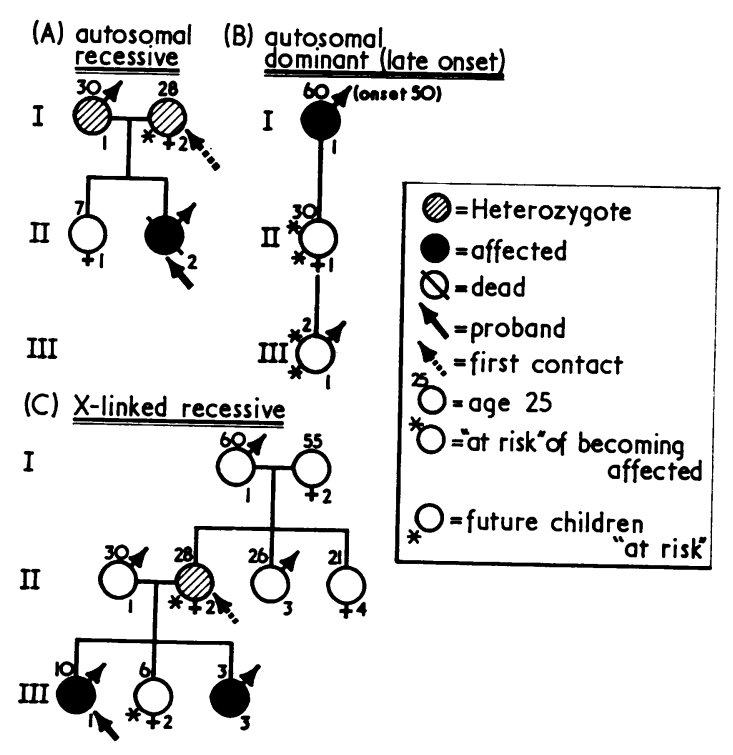

FIG. 1. Pedigrees illustrating the methods of assessing risks (see
text). 53 
affected son, the proband. The risk of the next child being affected is high $(25 \%)$ and this risk is allocated to the mother. There are no others at risk in the family.

In family $B$, suppose the autosomal dominant (AD) condition is Huntington's chorea. From a cumulative graph by onset age of cases of this disease (Emery, 1969), the risks of becoming affected are about $40 \%$ for the mother and $20 \%$ for the son; the risks of having children with the abnormal gene are then $20 \%$ and $10 \%$ respectively, so both are deemed to be also at risk of having children who may become affected. Since the mother (II.1) has been known to be at risk since her father's diagnosis some 10 years ago the birth of her 'at risk' son could have been prevented.

A more complex case (family $\mathrm{C}$ ) is illustrated for an $\mathrm{X}$-linked recessive (XR) condition, eg, Duchenne muscular dystrophy. With two affected sons, the mother is a definite carrier. The risks of her next having an affected boy or carrier daughter are each $25 \%$. These risks have been summed in the XR conditions so that the combined risk in this case is taken as $50 \%$. Similarly the daughter (III.2) is taken to be at risk since she has a $50 \%$ chance of being a carrier. Assuming the proband was diagnosed early, the birth of his affected brother (III.3) might have been prevented. Is the contact's sister (II.4) at risk ? The probability that the grandmother (I.2) is a carrier is $33 \%$, so the sister (II.4) has a $17 \%$ chance of being a carrier. Her risk of having an affected son or a carrier daughter is thus $18 \%$. Further information on her carrier status could be obtained by a serum creatine kinase test (eg, Fig. 2) leading to a more precise estimate of her risk (see Emery, 1969).

Material. The families studied were from either the Edinburgh or the Manchester region during 1965-70. Many of the families were referred for genetic counselling, but some were seen for other reasons: diagnosis, teaching purposes, or research work. Some of the families were traced from hospital or health department records or through members of certain societies (eg, Muscular Dystrophy Group). The kinds and frequency of the diseases studied thus reflect the work and interests of this Department rather than the spectrum of genetic disease in the population. No attempt was made to ascertain all cases of genetic disease in a region so that this is essentially a study of ascertained families rather than a population study.

Details about the family were usually obtained from the first person to be seen in the family, the first contact. This verbal report about the pedigree and the ages and disease status of family members provided most of the information on which the following analyses are based. To systematize the form of information collected, a special record card was designed (Fig. 2) and this was used for coding and punching the data on punch cards. Details on name, address, general practitioner, etc were recorded. At examination the clinician gave a summary of the clinical report and findings, filled in other relevant details and circled the appropriate responses. Each family was allocated a separate family number. Separate cards were completed $\frac{\$}{\mathbb{\Phi}}$ for any further members of the family who were judged $\Omega$ to be at risk. A special two-part disease code was de- $\mathbb{D}$ veloped but, to conform with common usage, the Inter- $\mathbb{D}$ national Disease Classification (with an additional 5th digit to allow for discrimination between different $\stackrel{\vec{s}}{\rightarrow}$ genetic conditions) will be used in future. A second $\overline{0}$ disease category was available, to record associations $\frac{C}{O}$ of different diseases in one family. The remainder of $\frac{\bar{\omega}}{\bar{\omega}}$. the first side of the card dealt with details on sex, dates of $\bar{\nabla}$ birth, details of the visit, and the mode of referral.

On the reverse side of the card (Fig. 2 below), the कै pedigree and further details were recorded as shown. $\vec{\circ}$
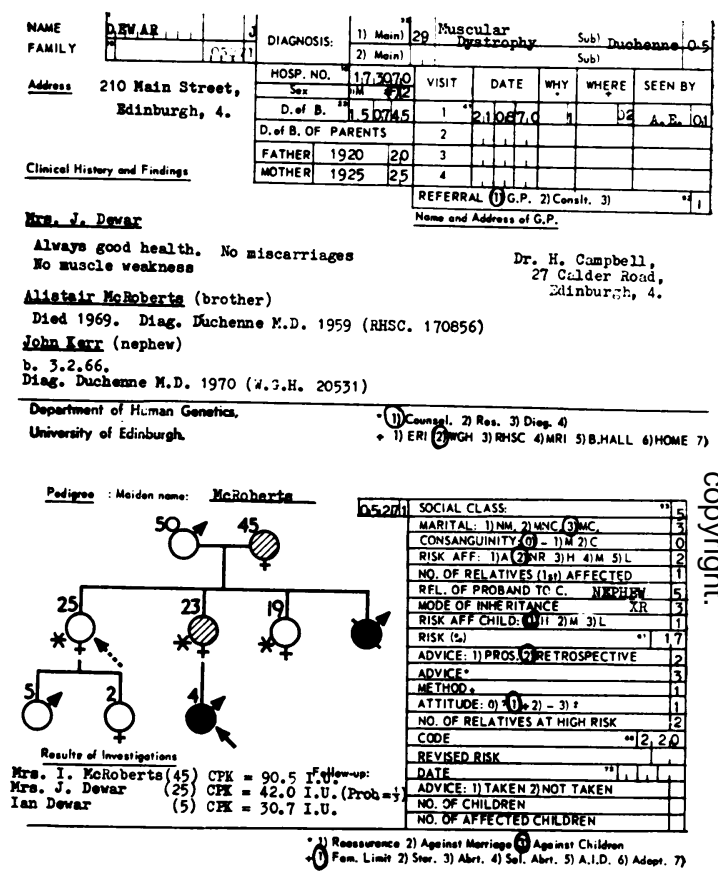

FIG. 2. Example of a completed family record card. The details are purely fictitious and are used only for illustration.

These included social class ( 5 groups); marital status (not married, married with no children, married with children); consanguinity (none, mild, close); status (affected, no risk, high risk, medium risk, low risk); number of first-degree relatives affected; relationship of the proband; mode of inheritance; risk of having an affected child (high, medium, low-and as a percentage); and whether the record was made before (prospective) or after (retrospective) the birth of an affected or 'at risk' child to the individual concerned.

If the person was counselled, the advice given was noted, together with any method of family limitation advised and the person's attitude to the counselling. Finally, for the first contact in the family, the number and relationship codes of relatives at risk were recorded. 
Further columns were left for any revision of the risks and for details of follow-up.

The information obtained on the families was not always complete because the cards for relatives at risk were prepared from the information given by the first contact at interview. Moreover some of the families were ascertained before the present recording scheme was developed, so past files had to be used and these were often incomplete. This may have led to some underestimation of the numbers affected and at risk, for only recorded information could be used. For analysis on a particular topic all data available on that topic were used. Almost all families ascertained were included in the analysis. These included conditions that were either not serious or not genetic or whose nature was not resolved, and form the 'other' category in Table I. The term 'multifactorial inheritance' in Table I refers to familial diseases which are possibly due to many loci plus the effects of environment and include some of the congenital malformations, diabetes mellitus, and schizophrenia (Carter, 1969).

\section{Results}

Families. Reasons for ascertainment and other details about the families studied are given in the series of histograms in Fig. 3. Over half of the families were referred for genetic counselling and the rest mainly for diagnosis or research. The chief source of families referred was the hospital consultant who contributed almost two thirds of the total. Most of the remainder were referred directly by the family's general practitioner. The distribution of the families by social class, though not directly comparable to the distribution in the
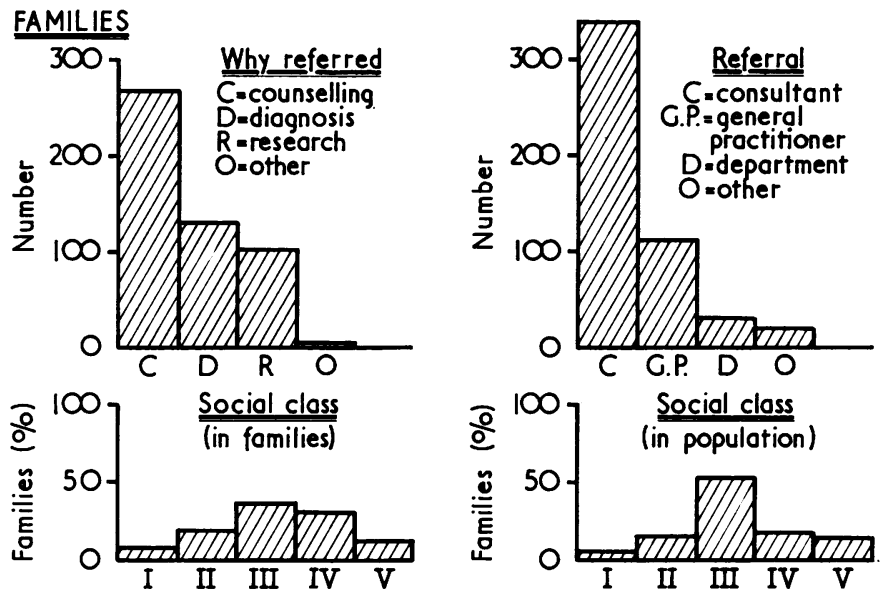

ALL FIRST CONTACTS
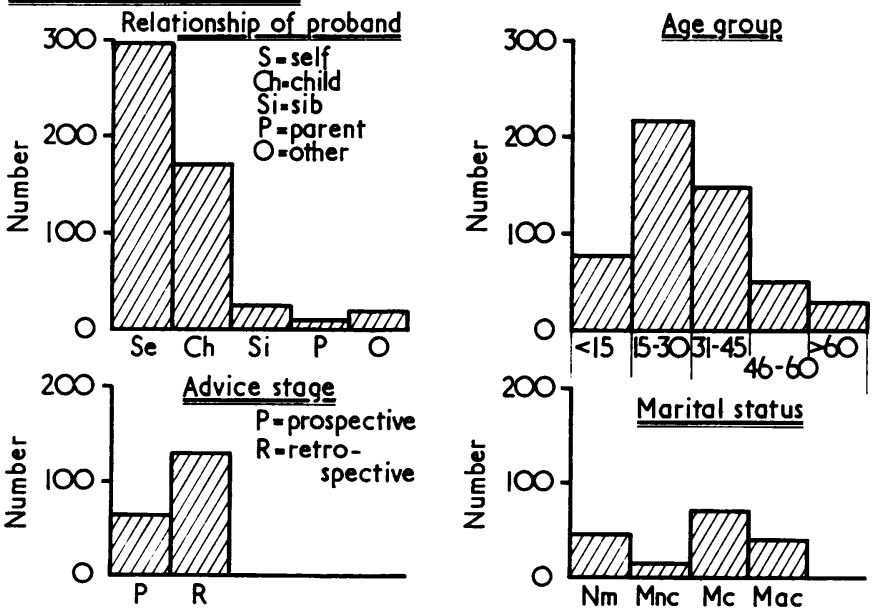

FIG. 3. Distributions of families and of first contacts (for serious genetic conditions) by various classifications of the data. 
population (1961 Census) because of age and other differences, shows an apparent excess of families in social classes 1 and 4 and a corresponding deficit in class 3. However all social classes are well represented in the families ascertained.

First Contacts. The remaining histograms in Fig. 3 refer specifically to the first contact in each family. Most of these were either the affected proband (usually in families referred for diagnosis or research) or the proband was the child of the first contact (usually in families referred for genetic counselling). This indicated that few other relatives in affected families were referred about their possible risk ( $c f$, Fig. 6). The age distribution of first contacts shows that they were largely in the reproductive age groups. This reflects concern about their risks of having affected children and about affected children born. Two thirds of the contacts at risk of having affected children were seen retrospectively, that is after the birth of an affected or at risk child. Among first contacts who were married and at risk of having affected children, almost $40 \%$ had no children (MNC) or had only affected children (MAC) so far. Genetic counselling was thus very relevant to them at this stage in their family life.

Risks and Mode of Inheritance. A tabulation by mode of inheritance of the numbers affected and the numbers at risk is given in Table I. In some 114 of the families recorded, the disease was not serious or judged to be not genetic. No further persons in these families were considered to be at risk. Among the families with chromosomal abnormalities or with multifactorial inherited conditions, there were relatively few persons at risk. By contrast, for diseases inherited in a simple Mendelian manner, a high proportion of the families had persons at risk; autosomal dominant (AD) $90 \%$, autosomal recessive (AR) $53 \%, X$-linked recessive (XR) $84 \%$. This confirms theoretical calculations (Smith, 1970) that preventive methods will be most effective for the simply inherited genetic diseases.

The numbers affected reflect the burden of the genetic conditions on these families. There was an average of over three persons affected per family for the AD conditions, two persons for the XR conditions, and about 1.5 persons for the $A R$ and multifactorial conditions. Moreover, the burden to the family is a continuing one in that a high proportion (over two thirds) of the affected persons are still alive.

The future prospects for these families are also serious because many have further members at risk either of becoming affected themselves or of having affected children. The distribution of the numbers at risk is shown in Fig. 4. Half the families with persons at risk had more than one member at risk and some families had many members at riskIn Table I, the three categories listed under 'numberat risk' are mutually exclusive so their total indicates the total number at risk in these families. This averages 4.0 persons for $\mathrm{AD}$ conditions, 3.5 persons

TABLE I

MODE OF INHERITANCE OF NUMBERS AFFECTED AND NUMBERS AT RISK IN 559 FAMILIES ASCERTAINED

\begin{tabular}{|c|c|c|c|c|c|c|}
\hline & \multicolumn{5}{|c|}{ Serious Genetic Conditions } & \multirow{3}{*}{ Others* } \\
\hline & \multicolumn{5}{|c|}{ Mode of Inheritance } & \\
\hline & $\begin{array}{c}\text { Autosomal } \\
\text { Dominant } \\
\end{array}$ & $\begin{array}{c}\text { Autosomal } \\
\text { Recessive } \\
\end{array}$ & $\begin{array}{c}\text { X-linked } \\
\text { Recessivet }\end{array}$ & $\begin{array}{c}\text { Multi- } \\
\text { factorial }\end{array}$ & $\begin{array}{c}\text { Chromo- } \\
\text { somal }\end{array}$ & \\
\hline $\begin{array}{l}\text { Families } \\
\text { Number } \\
\text { Number with someone at risk } \neq \\
\end{array}$ & $\begin{array}{l}124 \\
111 \\
\end{array}$ & $\begin{array}{r}112 \\
59 \\
\end{array}$ & $\begin{array}{r}102 \\
86 \\
\end{array}$ & $\begin{array}{l}78 \\
18 \\
\end{array}$ & $\begin{array}{r}29 \\
2 \\
\end{array}$ & $\begin{array}{r}114 \\
0\end{array}$ \\
\hline $\begin{array}{l}\text { Persons } \\
\text { Number affected } \\
\text { All } \\
\text { Alive } \\
\text { Number at risk } \\
\text { Only of becoming affected } \\
\text { Both of becoming affected and of having } \\
\text { affected children** } \\
\text { Only of having affected children }{ }^{* *}+\dagger \\
\end{array}$ & $\begin{array}{r}361 \\
255 \\
\\
39 \\
239 \\
158 \\
\end{array}$ & $\begin{array}{r}157 \\
119 \\
5 \\
0 \\
54\end{array}$ & $\begin{array}{r}198 \\
137 \\
15 \\
15 \\
272 \\
\end{array}$ & $\begin{array}{r}113 \\
68 \\
4 \\
2 \\
16 \\
\end{array}$ & $\begin{array}{r}32 \\
24 \\
0 \\
0 \\
2 \\
\end{array}$ & $\begin{array}{r}90 \\
72 \\
0 \\
\\
0 \\
0 \\
\end{array}$ \\
\hline $\begin{array}{l}\text { Births since } 1.960 \text { at risk a priori } \\
\text { Number of children } \\
\text { Affected } \\
\text { At riskt† } \\
\text { Not at risk }\end{array}$ & $\begin{array}{r}23 \\
77 \\
8\end{array}$ & $\begin{array}{r}14 \\
2 \\
15\end{array}$ & $\begin{array}{l}21 \\
77 \\
12\end{array}$ & $\begin{array}{l}3 \\
3 \\
1\end{array}$ & $\begin{array}{l}\mathbf{0} \\
\mathbf{0} \\
\mathbf{0}\end{array}$ & $\begin{array}{l}0 \\
0 \\
0\end{array}$ \\
\hline
\end{tabular}

* Not serious, not resolved, not genetic. +93 Families with muscular dystrophy. $¥$ Risk $10 \%$ or higher. ** Persons under age 40. t† Includes carrier daughters in X-linked disorders. (For other details see text.) 


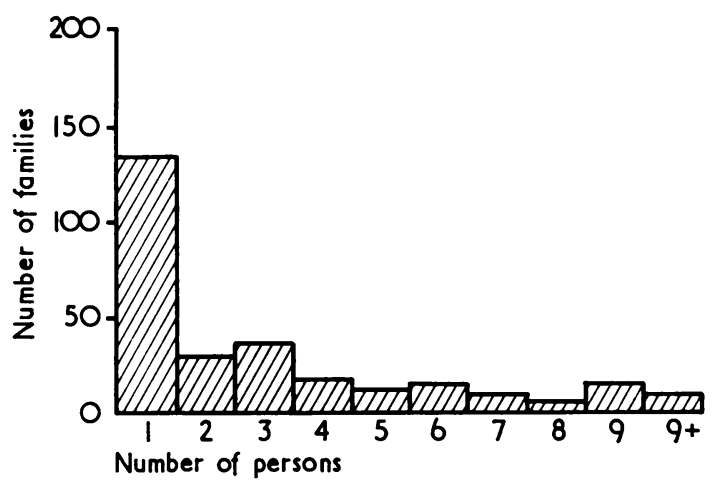

FIG. 4. Distribution of families by the number of persons at risk.

for XR conditions, and about one person for AR and multifactorial conditions.

Individuals at Risk. People at risk of becoming affected were largely in families with $\mathrm{AD}$ conditions with late onset (Table I), where a parent becomes affected after his children (and even grandchildren) have been born. With increasing age the risks to the children (if still unaffected), and to the grandchildren, will gradually fall. These patterns are reflected in the distribution by age of the numbers at risk of becoming affected (Fig. 5).

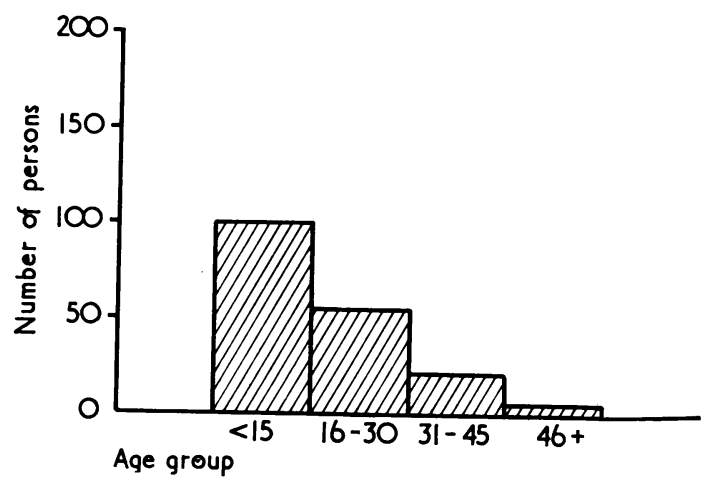

FIG. 5. Distribution by age of persons at risk of becoming affected.

For individuals at risk of having affected children or children at risk, all three simple modes of inheritance ( $A D, A R$, and $X R$ ) were involved, although the $\mathrm{AD}$-inherited conditions again predominated. The distribution of risks by age is given in Table II. The risks fall naturally into 3 groups; at about $50 \%$, at around $25 \%$, and from 10 to $19 \%$. The latter group represent only $14 \%$ of all at risk, so it is the higher risk categories which predominate. The distribution of the risks also changes with age $\left(\chi^{2}=35, \mathrm{p}<0.001\right)$ with the proportion in the highest risk category increasing with age. This was largely because such high risks occur firstly, in $\mathrm{AD}$ conditions when the person is diagnosed as affected and onset is often late and secondly, in XR conditions when a mother is proven to be a carrier after the birth of an affected son.

\section{TABLE II}

\begin{tabular}{|c|c|c|c|}
\hline \multirow{2}{*}{ Age (yr) } & \multicolumn{3}{|c|}{ Percentage Risk } \\
\hline & $10-19 \%$ & $20-29 \%$ & over $30 \%$ \\
\hline $\begin{array}{l}\text { Under } 16 \\
6-30 \\
1-40\end{array}$ & $\begin{array}{l}29 \\
36 \\
19\end{array}$ & $\begin{array}{r}122 \\
94 \\
44\end{array}$ & $\begin{array}{l}41 \\
98 \\
60\end{array}$ \\
\hline
\end{tabular}

The distribution by relationship of the proband to those at risk in the family is given in Fig. 6 . There is a strong contrast with the equivalent distribution for first contacts (Fig. 3). This demonstrates that many kinds of relatives who are at high risk are not being ascertained or counselled by normal medical practice.

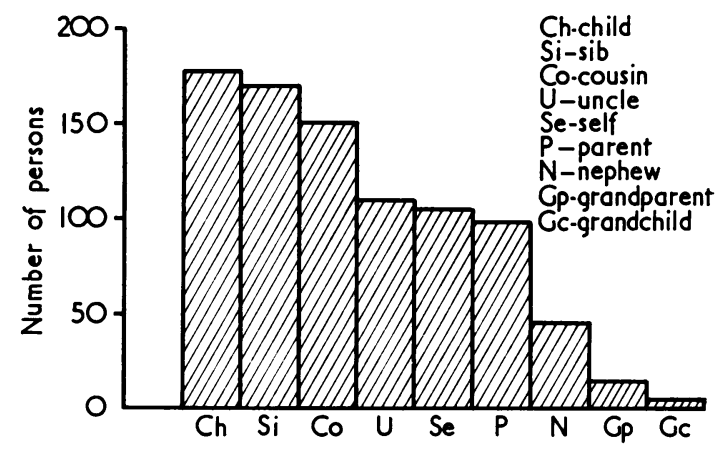

FIG. 6. Distribution of persons at risk of having affected children, by relationship of the proband.

Births at Risk a priori. The results for children born since 1960 to parents who where at risk a priori of having affected children confirm the risks involved. Of 256 births in this category, some 61 $(24 \%)$ of the children were affected while a further $159(61 \%)$, though normal so far, are still at risk of becoming affected themselves or of having affected children. 


\section{Discussion}

In this department a genetic register system is being developed and is referred to by the acronym 'RAPID' (Register for the Ascertainment and Prevention of Inherited Disease). Here we report results for the initial stage of development of the register, using some 559 ascertained families. The objectives in this stage were (1) to gain experience in recording and handling family data and in assessing risks to family members and (2) to examine the need and scope for a preventive system in practice. Our results clearly show the area where preventive effort can be best applied and justify the implementation of a genetic register system in practice.

The main scope for preventing genetic disease lies, at present, with the simply inherited diseases despite the fact that other diseases are much more frequent in the population. This is because the proportion of individuals at risk is greater, and the risks are higher in families with simply inherited conditions than in families with multifactorial or chromosomal disorders. Thus it is proposed that preventive effort should be largely restricted, at least initially, to the simply inherited conditions. Thus we have defined a discrete problem area which should give worthwhile returns for any resources committed. However, it should be emphasized that even for the simply inherited disorders, it will be possible to prevent only a proportion of cases, since some will occur in families which have not been ascertained previously (Smith, 1970).

In the 338 families with simply inherited conditions, there were some 716 affected individuals, of whom 511 are still alive. This emphasizes the past and continuing burdens on these families and their need for medical care and supervision. Moreover, and perhaps more disconcerting, is the fact that the burden in these families is likely to increase in that there are some 797 individuals still at risk, either of becoming affected themselves or of having children who may be affected. This shows the need for genetic counselling and supervision to be extended to all family members at risk (Fig. 6), rather than only to the first contact in the family. That is, the initiative in prevention should be undertaken as a health service, rather than left to the individual who may be unaware of his risk. This point was stressed in a previous study (Emery and Smith, 1970) where it was shown that only a small proportion (14\%) of those at risk was referred for genetic counselling. There is at present no defined procedure or responsibility for tracing and counselling individuals known to be at risk. Herein lies the value of a genetic register system.
The development of the RAPID system is now proceeding in several areas. One is to follow-up individuals who have been counselled, to keep in touch with the family, and to assess the value of the counselling methods in preventing genetic disease. Another is to develop procedures for contacting and counselling others at risk in the family, working always through the general practitioner of the person concerned. To extend the system to a population basis, methods and sources for ascertaining families with simply inherited genetic disease are being examined. Through linkage with hospital, GP, health department, and other records, relevant families will be screened for investigation and counselling. The data storage and handling operations are being organized around a computer, and much of the system software has been written. The procedures include data vetting, monitoring, listing, updating, scheduling for follow-up, and other items. Several hurdles are foreseen. Among them are the geographical dispersion of family members, security of data on file, the privacy of the individual, the attitude of healthy individuals to possible genetic risks, the effectiveness of counselling, and the organizational details required to make the register an effective preventive system.

\section{Summary}

This report concerns data on 559 families with genetic disease referred for genetic counselling, diagnosis or research. It summarizes our work on the initial stages in the development of a genetic register system (RAPID) for the ascertainment and prevention of genetic disease. The procedures for assessing risks to individuals in ascertained families are described, and methods of recording and handling the relevant family records and details on individuals 'at risk' are also shown.

Data are presented on reasons and routes of referral and on social class of the families ascertained and on the age, marital status, risk etc of individuals referred. In the $\mathbf{5 5 9}$ families studied there were 951 affected individuals (of whom $70 \%$ were still alive) and some 821 individuals who were judged to be at risk themselves or at risk of having affected children. The analyses confirm the need for a genetic register system in practice and show that the simply inherited genetic diseases offer the best scope in prevention.

We would like to thank Mrs E. R. Clack and Miss M. S. Watt, SRN for their help in tracing families. This 
work was supported by grants from the Scottish Hospital Endowments Research Trust and the Muscular Dystrophy Group of Great Britain.

\section{REFERBNCES}

Carter, C. O. (1969). Genetics of common disorders. British Medical Bulletin, 25, 52-57.

Emery, A. E. H. (1969). Genetic counselling. Scottish Medical fournal, 14, 335-347.

Emery, A. E. H. and Smith, C. (1970). Ascertainment and prevention of genetic disease. British Medical fournal, 3, 636-637.
Fraser, G. R., and Motulsky, A. G. (1968). Genetic effects of selective abortion'for inherited diseases. American fournal of Human Genetics, 20, 489.

McKusick, V. A. (1969). Family-oriented follow-up. fournal of Chronic Diseases, 22, 1-7.

Roberts, D. F., Chavez, J., and Court, S. D. M. (1970). The genetic component in child mortality. Archives of Disease in Childhood, 45, 33-38.

Roberts, J. A. (1962). Genetic prognosis. British Medical fournal, 11, 578-592.

Smith, C. (1970). Ascertaining those at risk in the prevention and treatment of genetic disease. In Modern Trends in Human Genetics, vol. 1, pp. 350-369, ed. by A. E. H. Emery. Butterworth, London. 